\title{
Two-electron-satellite transition of donor bound exciton in ZnO: Radiative Auger effect
}

\author{
X. H. Wang and S. J. Xua) \\ Department of Physics and HKU-CAS Joint Laboratory on New Materials, The University of Hong Kong, \\ Pokfulam Road, Hong Kong, China
}

(Received 20 March 2013; accepted 26 April 2013; published online 10 May 2013)

\begin{abstract}
Two-electron-satellite (TES) transition of donor bound exciton $\left(\mathrm{D}_{0} \mathrm{X}\right)$ is an interesting many-body quantum process. In this letter, precise luminescence spectra and temperature behaviors of the TES transition of aluminum bound exciton in two kinds of zinc oxide single crystals were investigated in detail. It is found that the TES transition can be treated as a radiative Auger process in which the temperature dependence of the emission intensity of the transition can be fitted very well with a model taking into account the temperature dependent Auger term and thermal dissociation of the

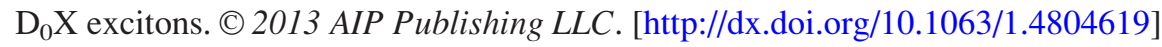

Recombination of excited or injected excess carriers in semiconductors is one kind of fundamental quantum process. It can be either radiative or non-radiative. The former gives light emission while the latter does not. Two types of important non-radiative processes, namely, Shockley-Read-Hall (SRH) process and Auger process, have been identified and documented. ${ }^{1}$ However, one peculiar radiative Auger effect, i.e., a simultaneous emission of a light-quantum and an electron by the excited atom in the $K$ x-ray spectra observed by Bloch and Ross in molybdenum, rhodium, etc, ${ }^{2}$ or a neutral donor bound exciton recombines radiatively with simultaneous elevation of the second electron to various excited states of the donor and even into the conduction band of host material observed by Nelson et al. in indirect gap semiconductors such as $\mathrm{GaP}$ and $\mathrm{Si}^{3}{ }^{3}$ seems more exciting due to the involvement of many quanta. They also pointed out that such radiative Auger effect should predominate in the decay of excitons bound to neutral donors or acceptors for a wide variety of semiconductors. Recently, there has been strong interest to Auger effects in various semiconductors and nitride based light emitting devices, ${ }^{4-7}$ since it is regarded as an important mechanism for light emission efficiency droop. Being as a wide gap polar semiconductor having outstanding physical properties and potential applications in optoelectronics and photonics, zinc oxide $(\mathrm{ZnO})$ has received a great deal of interest in past decades. Meanwhile, with wellestablished growth technique for high quality single crystals, it offers an ideal platform to study various excitonic transitions and exciton-phonon interactions. ${ }^{8-12}$

In high quality $\mathrm{ZnO}$ crystals, direct radiative recombination of shallow donor bound exciton $\left(\mathrm{D}_{0} \mathrm{X}\right)$ is usually dominant in photoluminescence (PL) spectra at cryogenics temperatures. ${ }^{8-10}$ However, so-called two-electron-satellite (TES) transition is still an important recombination channel for the annihilation of $\mathrm{D}_{0} \mathrm{X} .{ }^{13}$ As a result of this kind of recombination, several weak emission lines can be seen on the low energy side of the $\mathrm{D}_{0} \mathrm{X}$ principal line. As mentioned earlier, TES transition was first discussed in some indirect

\footnotetext{
${ }^{\text {a) }}$ Author to whom correspondence should be addressed. Electronic mail: sjxu@hku.hk
}

gap semiconductors such as $\mathrm{GaP}$ and $\mathrm{Si}^{3,14}$ later in some direct gap semiconductors such as GaAs, ${ }^{15} \mathrm{InP},{ }^{16}$ and wide band gap GaN. ${ }^{17}$ These studies firmly show that TES radiative transition is a quite general quantum process in a wide variety of semiconductors. In fact, such a peculiar quantum transition in which a few particles are involved carries rich information on impurity, exciton, exciton-impurity electron coupling, and even exciton-phonon coupling. Therefore, a deep understanding of the TES transition in wide gap semiconductors like $\mathrm{ZnO}$ is highly desirable and timely required.

In this letter, we present a detailed investigation of the TES emission lines from $\mathrm{ZnO}$ bulk crystals grown with two major growth techniques as a function of temperature. It is found that the TES transition and its temperature dependence can be interpreted well in term of the radiative Auger transition. Moreover, in sharp contrast with the $\mathrm{D}_{0} \mathrm{X}$ principal emission line, the TES emission intensity shows a distinct dependence on temperature, i.e., a first increase in intensity with increasing temperature and then decrease.

The $\mathrm{ZnO}$ samples used in this study were high quality single crystals with c-cut plane (0001) orientation grown by melt-grown method (Cermet, Inc.) and hydrothermal method (Tokyo Denpa, Inc.). For variable-temperature PL measurements, the samples were mounted on the cold finger of cryostat (Oxford Instruments) with temperature varying from $1.5 \mathrm{~K}$ to $300 \mathrm{~K}$. The excitation light source was a Kimmon He-Cd UV Laser with an emission wavelength of $325 \mathrm{~nm}$, and the optical signal was guided into a two-meter long monochromator (McPherson, Inc.) with optimal resolution of $0.003 \mathrm{~nm}$. PMT was used to detect the luminescence signal and convert it into an electrical signal while standard lock-in amplifier was employed to enhance the signal-tonoise ratio.

Figure 1 shows the semi-logarithmic PL spectra of the two samples at $1.5 \mathrm{~K}$. For both the $\mathrm{ZnO}$ samples grown with different methods, there are basically three structures in the $\mathrm{D}_{0} \mathrm{X}$ region. Among them, so-called $\mathrm{I}_{6}$ emission peak is dominant, which was ascribed to the emission line of aluminum bound exciton. ${ }^{18}$ The TES emission lines of $\mathrm{I}_{6}$ can be clearly seen at the lower energy side for the two samples. For the hydrothermal grown $\mathrm{ZnO}$ sample from Tokyo 


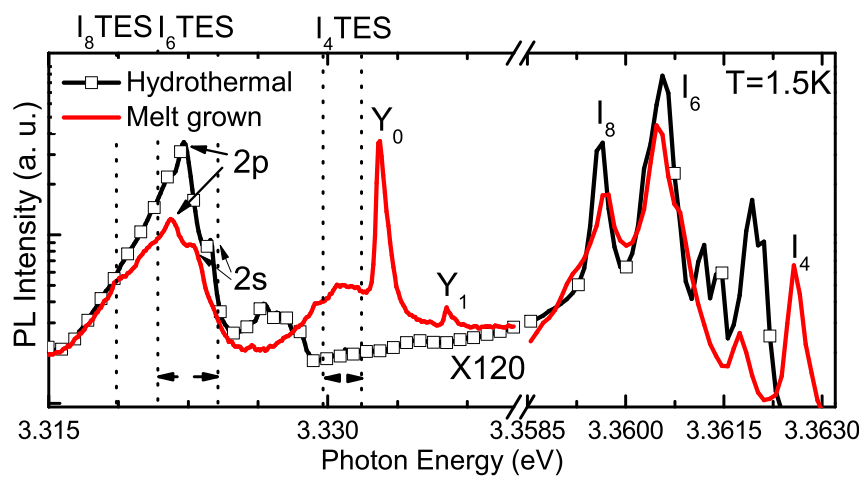

FIG. 1. Semi-logarithmic PL spectra of the two $\mathrm{ZnO}$ samples at $1.5 \mathrm{~K}$. The emission intensity in the TES region is manually magnified by 120 for clear comparison with the emission intensity $\mathrm{I}_{\mathrm{x}}$ of $\mathrm{D}_{0} \mathrm{X}$.

Denpa, however, the narrowest emission line $\mathrm{Y}_{0}$ is not observed that was previously investigated and suspected to be from some structural defect depending on growth method. ${ }^{19}$ Its nature is still unclear because such narrowest emission line was reported in other hydrothermal grown $\mathrm{ZnO}$ samples. ${ }^{20}$ In the present study, we concentrate on the TES transition of the dominant $\mathrm{D}_{0} \mathrm{X}\left(\mathrm{I}_{6}\right)$. It splits into double structures identified as the $2 \mathrm{~s}$ and $2 \mathrm{p}$ TES modes, as shown in Fig. 1. The splitting of the $2 s$ and $2 p$ states is due to the anisotropy of $\mathrm{ZnO}$ crystal field and the polar interaction owing to the LO phonon vibrations. A quantitative expression for the energy of $2 \mathrm{~s}$ and $2 \mathrm{p}$ states can be written as ${ }^{12}$

$$
\begin{gathered}
E_{2 s}=-\frac{R^{*}}{4}\left(1+A_{2 s}+P_{2 s}\right)-E_{c h}, \\
E_{2 p}=-\frac{R^{*}}{4}\left(1+A_{2 p}+P_{2 p}\right),
\end{gathered}
$$

where $R^{*}$ is the polaron effective Rydberg energy, while $E_{c h}$, $A_{2 s, 2 p}$, and $P_{2 s, 2 p}$ are all positive values which represent the chemical shift of $2 \mathrm{~s}$ state, anisotropy factor and the polar correction for $2 \mathrm{~s}$ and $2 \mathrm{p}$ states, respectively. Therefore, if the various corrections were neglected, the energy for $2 \mathrm{~s}$ and $2 \mathrm{p}$ states should be equal to $-R^{*} / 4$ with reference point set at the continuum. The corrections make the $2 \mathrm{~s}$ and $2 \mathrm{p}$ states shift towards lower energy. It should be noted that the $\mathrm{D}_{0} \mathrm{X}$ $\left(\mathrm{I}_{6}\right)$ lines and their respective first-order phonon replicas (not shown here) have the exact energetic locations for both the samples when the instrumental error is taken into account. However, a detailed inspection of the TES spectra of the two samples at different temperatures shows that the energetic locations of the TES lines exhibit unexpectedly large difference, i.e., the $2 \mathrm{p}$ and $2 \mathrm{~s}$ states in the melt-grown sample are 0.7 and $0.9 \mathrm{meV}$, respectively, lower than those of the thermal grown sample. Moreover, the energy separation $\left(E_{2 p}-E_{2 s}\right)$ between the two excited states is $1.46 \mathrm{meV}$ and $1.20 \mathrm{meV}$ for the thermal grown sample and melt grown sample, respectively. Such large energy difference for the TES between the two samples is rather unusual. From the parameters given in Refs. 12 and 17, it can be calculated that, for the 2 s state, the energy shift due to the polar interaction $P_{2 s}$ is two orders of magnitude larger than the anisotropic correction $A_{2 s}$. Thus, the energy correction for the $2 \mathrm{~s}$ state can be approximated as an outcome of polar interaction and chemical shift. In fact, the anisotropy factor has been argued to produce less significant correction compared to the polaron coupling. ${ }^{21,22}$ Further investigations are thus needed to clarify the physical mechanism causing the unusually large difference in energy for the TES lines of the same $\mathrm{D}_{0} \mathrm{X}$ between the two samples. Precise many-body interactions involving exciton, impurity electron, phonon, and even photon shall be considered for explaining the detailed structures in the PL spectra of $\mathrm{ZnO}{ }^{9}$

Figure 2 shows the variable temperature PL spectra of the two samples in the temperature range of 1.5-30.0 K. The PL intensity of the $\mathrm{D}_{0} \mathrm{X}$ principal line decreases rapidly with

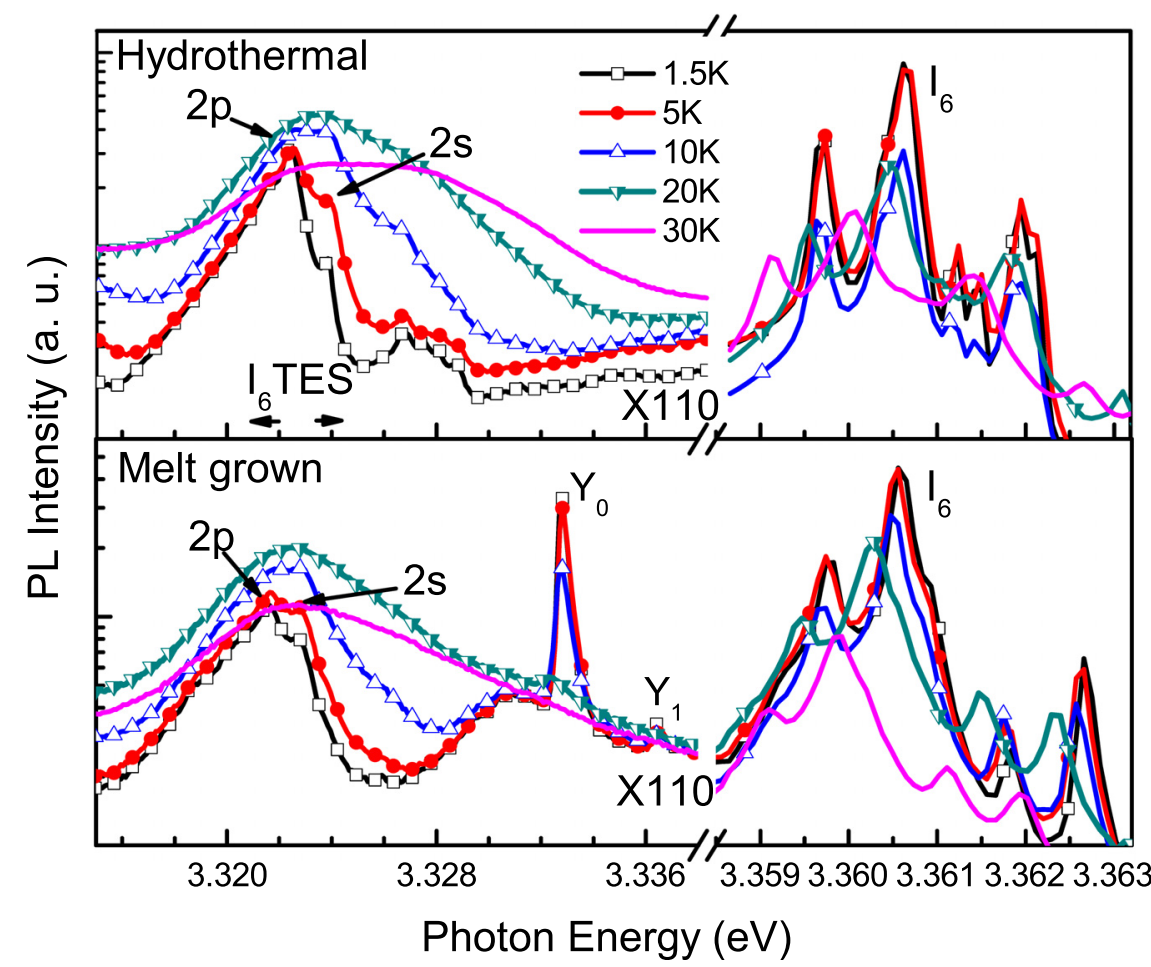

FIG. 2. Semi-logarithmic PL spectra of the two samples at different temperatures from 1.5 to $30 \mathrm{~K}$. The intensity of the TES lines is magnified by 110 for clarity. 
increasing temperature, accompanying with noticeable redshift and broadening, which are common features of the donor bound excitonic emission due to the thermal effects. ${ }^{9}$ However, the TES emissions of the $\mathrm{D}_{0} \mathrm{X}$ exhibit very different dependence on temperature. As temperature is increased from $1.5 \mathrm{~K}$ to $20 \mathrm{~K}$, the intensity of both the $2 \mathrm{~s}$ ad $2 \mathrm{p}$ emission peaks increases, especially for the $2 \mathrm{~s}$ emission whose intensity rises rapidly and even becomes stronger than the $2 p$ emission at $20 \mathrm{~K}$. However, when temperature is up to $30 \mathrm{~K}$, the TES emission peak experiences a significant decrease in intensity and simultaneously broadening in width, which is ascribed to the substantial thermal dissociation of the $\mathrm{D}_{0} \mathrm{X}$ excitons.

In Fig. 3, a more quantitative view for the temperature dependency of various radiative channels of $\mathrm{D}_{0} \mathrm{X}$ is presented by plotting the integrated intensity (symbols) versus temperature along with the fitting curves (solid lines) of the $\mathrm{D}_{0} \mathrm{X}$ and TES data. In the low temperature range of $1.5-20.0 \mathrm{~K}$, the $\mathrm{D}_{0} \mathrm{X}$ and its TES exhibit very different dependence on temperature for both the samples. That is, the $\mathrm{D}_{0} \mathrm{X}$ intensity monotonically decreases while the TES emission increases with increasing temperature. As mentioned earlier, the rapid drop of the $\mathrm{D}_{0} \mathrm{X}$ emission intensity is mainly due to the efficient thermal dissociation of $\mathrm{D}_{0} \mathrm{X}$ excitons (i.e., to free excitons (FE)) since the FE intensity shows a substantial increase (not shown here). Therefore, to fit the temperature dependence of the $\mathrm{D}_{0} \mathrm{X}$ emission intensity, only the thermal dissociation factor is considered and thus the well-known one-channel dissociation Arrhenius model was used ${ }^{23}$

$$
\frac{I(T)}{I_{T=0}}=\frac{1}{1+A \times \exp \left(-\frac{E_{a}}{k_{b} T}\right)},
$$

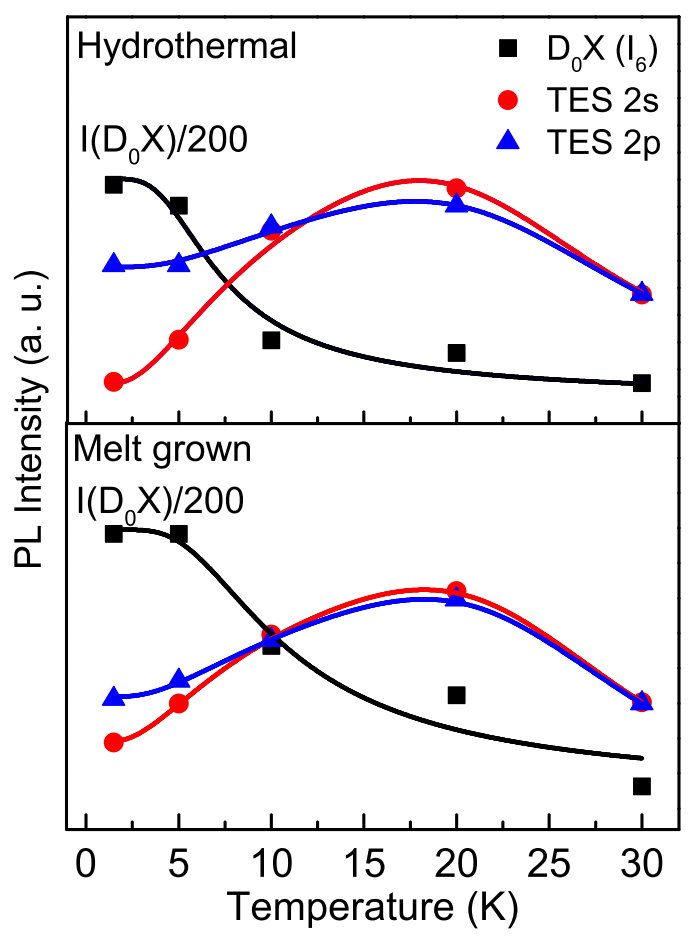

FIG. 3. Integrated PL intensity of the $D_{0} X$ and TES transitions as a function of temperature. The solid curves represent theoretical fitting for the corresponding experimental data. The intensity of $\mathrm{D}_{0} \mathrm{X}$ is divided by 200 for convenience of comparison. where $E_{a}$ is the dissociation energy of the $\mathrm{D}_{0} \mathrm{X}$ excitons, $A$ is a temperature independent factor, $k_{b}$ is the Boltzmann constant, and $T$ is the absolute temperature. From Fig. 3, it can be seen that the classic Arrhenius model works very well for the $\mathrm{D}_{0} \mathrm{X}$ principal transitions of the two samples. As for the TES transition, as viewed from Fig. 3, it shows a nonmonotonic dependence on temperature. For the temperature below $20 \mathrm{~K}$, the TES emission intensity unusually increases with increasing temperature. When the temperature is beyond $20 \mathrm{~K}$, it starts to decrease. Such distinct evolution with temperature suggests that the TES transition has very different mechanism compared with its parent transition (the $\mathrm{D}_{0} \mathrm{X}$ principal transition). Therefore, in order to find a quantitative description on the temperature dependence of the TES transition in the interested temperature range from 1.5 to $30.0 \mathrm{~K}$, we shall consider more factors such as temperature dependent radiative rate of the TES transition, in addition to influence of the thermal dissociation of the $\mathrm{D}_{0} \mathrm{X}$ excitons.

We start our discussion from a general definition of luminescence intensity, i.e., a product of total number and average rate of excitons involved in radiative process:

$$
I=N \cdot \frac{1}{\tau}
$$

where $N$ denotes the total number of $\mathrm{D}_{0} \mathrm{X}$ excitons involved in the radiative TES transition, and $1 / \tau$ is the average rate of the TES transition. For temperature dependence of $N$, it can be described with the Arrhenius model as above used. For the TES transition, it has been argued to be analogous to the radiative Auger effect observed in the x-ray photo-emission spectra, in which the electron is excited into continuum or bound states whereas an x-ray photon is emitted. The Auger transition rate in semiconductors was suggested to be exponentially dependent on temperature, say $e^{\left(-E / k_{B} T\right)} \cdot{ }^{24}$ In fact, such an exponential dependence has been further developed and modified for various band-to-band processes. ${ }^{4}$ In the case of the TES transition discussed here, we adopt following express to describe the temperature dependence of the TES transition rate:

$$
\frac{1}{\tau} \propto 1+B \times \exp \left(-\frac{\Delta E_{2 s(2 p)}}{k_{b} T}\right),
$$

where $B$ is a temperature independent factor, $\Delta E_{2 s(2 p)}$ represents a characteristic energy for the TES $2 \mathrm{~s}(2 \mathrm{p})$ state, respectively. For $\Delta E_{2 s(2 p)}$, it is equal to the electron transition energy from $1 \mathrm{~s}$ to $2 \mathrm{~s}(2 \mathrm{p})$ minus the energy difference between the quasi Fermi levels of $1 \mathrm{~s}$ and $2 \mathrm{~s}(2 \mathrm{p})$ state, i.e.,

$$
\Delta E_{2 s(2 p)}=\left(E_{2 s(2 p)}-E_{1 s}\right)-\left(E_{f 2 s(2 p)}-E_{f 1 s}\right),
$$

where $E_{f 2 s(2 p)}$ and $E_{f 1 s}$ are the quasi Fermi levels for 2s (2p) and $1 \mathrm{~s}$ state. Thus, by combining the temperature dependence of the exciton number and TES transition rate, the luminescence intensity formula of the radiative TES transition is yielded as

$$
I=N \cdot \frac{1}{\tau} \propto \frac{1+B \times \exp \left(-\frac{\Delta E_{2 s(2 p)}}{k_{b} T}\right)}{1+A^{\prime} \times \exp \left(-\frac{E_{a}^{\prime}}{k_{b} T}\right)} .
$$


TABLE I. Fitting parameters for the integrated PL intensity of the $\mathrm{D}_{0} \mathrm{X}$ and TES with Eqs. (3) and (7). The energy is given in the unit of meV.

\begin{tabular}{lll}
\hline \hline Sample & $\mathrm{D}_{0} \mathrm{X}\left(A, E_{a}\right)$ & \multicolumn{1}{c}{$\mathrm{TES}\left(B, \Delta E_{2 s(2 p)}, A^{\prime}, E_{a}^{\prime}\right)$} \\
\hline Hydrothermal & $9.99,1.71$ & $2 \mathrm{~s}: 10.03,0.91,126.52,11.60$ \\
& & $2 \mathrm{p}: 1.24,1.46,120.63,12.25$ \\
Melt grown & $6.23,2.18$ & $2 \mathrm{~s}: 72.67,0.88,194.19,13.26$ \\
& & $2 \mathrm{p}: 1.57,1.20,183.68,13.27$ \\
\hline
\end{tabular}

As shown in Fig. 3, the fitting curves (solid lines) according to Eqs. (3) and (7), respectively, show good agreement with experimental data of the $\mathrm{D}_{0} \mathrm{X}$ principal transition and the TES transition. The values adopted for the fitting parameters are listed in Table I. Although we are not able to calculate the exact values for $E_{f 2 s(2 p)}$ and $E_{f 1 s}$, the $\Delta E_{2 s(2 p)}$ fitting results are in a reasonable range, for example, the difference between $\Delta E_{2 p}-\Delta E_{2 s}$ is comparable to $E_{2 p}-E_{2 s}$ obtained from Fig. 1. Meanwhile, the fitting results for TES $2 \mathrm{~s}$ and $2 \mathrm{p}$ states in each sample show good consistence, i.e., the value of $E_{a}^{\prime}$ much larger than $E_{a}$, which means largely delayed thermal influence on the TES transition, as observed.

To conclude, we present a detailed study on the TES transition for the two commercial $\mathrm{ZnO}$ single crystals prepared with two typical main growth methods. Through analyzing the temperature dependence of the TES emission intensity, we show that the TES transition can be treated as a radiative Auger transition rather than a common radiative recombination like the direct radiative recombination of bound excitons. We believe that this work and view offer an important insight into the nature of satellite transitions of bound exciton complexes.

This work was financially supported by the Hong Kong RGC-GRF Grant (Grant No. HKU703612P) and partially by a grant from the University Grants Committee Areas of
Excellence Scheme of the Hong Kong Special Administrative Region, China (Project No. [AoE/P-03/08]).

${ }^{1}$ T. Goudon, V. Miljanović, and C. Schmeiser, SIAM J. Appl. Math. 67, 1183 (2007).

${ }^{2}$ F. Bloch and P. A. Ross, Phys. Rev. 47, 884 (1935).

${ }^{3}$ D. F. Nelson, J. D. Cuthbert, P. J. Dean, and D. G. Thomas, Phys. Rev. Lett. 17, 1262 (1966).

${ }^{4}$ N. K. Dutta and R. J. Nelson, J. Appl. Phys. 53, 74 (1982).

${ }^{5}$ Y. C. Shen, G. O. Mueller, S. Watanabe, N. F. Gardner, A. Munkholm, and M. R. Krames, Appl. Phys. Lett. 91, 141101 (2007).

${ }^{6}$ K. T. Delaney, P. Rinke, and C. G. Van de Walle, Appl. Phys. Lett. 94, 191109 (2009).

${ }^{7}$ F. Bertazzi, M. Goano, and E. Bellotti, Appl. Phys. Lett. 97, 231118 (2010).

${ }^{8}$ See, for example, a recent review article and references therein, Ü. Özgür, Ya. I. Alivov, C. Liu, A. Teke, M. A. Reshchikov, S. Doan, V. Avrutin, S.-J. Cho, and H. Morkoç, J. Appl. Phys. 98, 041301 (2005).

${ }^{9}$ S. J. Xu, S. J. Xiong, and S. L. Shi, J. Chem. Phys. 123, 221105 (2005).

${ }^{10}$ C. C. Zheng, S. J. Xu, J. Q. Ning, Y. N. Chen, B. K. Li, J. N. Wang, and C. M. Che, J. Phys. Chem. A 116, 381 (2012).

${ }^{11}$ C. C. Zheng, S. J. Xu, J. Q. Ning, Y. N. Chen, F. Zhang, and C. M. Che, Appl. Phys. Lett. 100, 221105 (2012).

${ }^{12}$ M. Dvorak, S.-H. Wei, and Z. Wu, Phys. Rev. Lett. 110, 016402 (2013).

${ }^{13}$ B. K. Meyer, H. Alves, D. M. Hofmann, W. Kriegseis, D. Forster, F. Bertram, J. Christen, A. Hoffmann, M. Straßburg, M. Dworzak, U. Haboeck, and A. V. Rodina, Phys. Status Solidi B 241, 231 (2004).

${ }^{14}$ P. J. Dean, J. D. Cuthbert, D. G. Thomas, and R. T. Lynch, Phys. Rev. Lett. 18, 122 (1967).

${ }^{15}$ F. A. J. M. Driessen, H. G. M. Lochs, S. M. Olsthoorn, and L. J. Giling, J. Appl. Phys. 69, 906 (1991).

${ }^{16}$ P. J. Dean and M. S. Skolnick, J. Appl. Phys. 54, 346 (1983).

${ }^{17}$ P. P. Paskov, B. Monemar, A. Toropov, J. P. Bergman, and A. Usui, Phys. Status Solidi C 4, 2601 (2007).

${ }^{18}$ B. Hönerlage and U. Schröder, Phys. Rev. B 16, 3608 (1977).

${ }^{19}$ M. R. Wagner, G. Callsen, J. S. Reparaz, J.-H. Schulze, R. Kirste, M. Cobet, I. A. Ostapenko, S. Rodt, C. Nenstiel, M. Kaiser, A. Hoffmann, A. V. Rodina, M. R. Phillips, S. Lautenschläger, S. Eisermann, and B. K. Meyer, Phys. Rev. B 84, 035313 (2011).

${ }^{20}$ K. Johnston, M. O. Henry, D. McCabe, E. McGlynn, M. Dietrich, E. Alves, and M. Xia, Phys. Rev. B 73, 165212 (2006).

${ }^{21}$ J. Flohrer, E. Jahnke, and M. Porsch, Phys. Status Solidi B 91, 467 (1979).

${ }^{22}$ A. V. Rodina, M. Dietrich, A. Göldner, L. Eckey, A. Hoffmann, Al. L. Efros, M. Rosen, and B. K. Meyer, Phys. Rev. B 64, 115204 (2001).

${ }^{23}$ F. E. Williams and H. Eyring, J. Chem. Phys. 15, 289 (1947).

${ }^{24}$ H. Fröhlich and J. O’Dwyer, Proc. Phys. Soc., London Sect. A 63, 81 (1950). 